\title{
Cardiovascular Outcomes of PCSK9 Inhibitors: With Special Emphasis on Its Effect beyond LDL-Cholesterol Lowering
}

\author{
Dhrubajyoti Bandyopadhyay $\mathbb{D}^{1},{ }^{1}$ Kumar Ashish, ${ }^{2}$ Adrija Hajra, ${ }^{3}$ \\ Arshna Qureshi, ${ }^{4}$ and Raktim K. Ghosh ${ }^{5}$ \\ ${ }^{1}$ Internal Medicine, Mount Sinai St Luke's Roosevelt Hospital Center, New York, NY, USA \\ ${ }^{2}$ University of Texas MD Anderson Cancer Center, Houston, TX, USA \\ ${ }^{3}$ IPGMER, Kolkata, India \\ ${ }^{4}$ Department of Medicine, Lady Hardinge Medical College, New Delhi, India \\ ${ }^{5}$ Metro Health, Case Western Reserve University, Cleveland, OH, USA \\ Correspondence should be addressed to Dhrubajyoti Bandyopadhyay; drdhrubajyoti87@gmail.com
}

Received 10 November 2017; Revised 15 January 2018; Accepted 20 February 2018; Published 25 March 2018

Academic Editor: Maurizio Averna

Copyright (c) 2018 Dhrubajyoti Bandyopadhyay et al. This is an open access article distributed under the Creative Commons Attribution License, which permits unrestricted use, distribution, and reproduction in any medium, provided the original work is properly cited.

\begin{abstract}
PCSK9 inhibitors, monoclonal antibodies, are novel antihypercholesterolemic drugs. FDA first approved them in July 2015. PCSK9 protein (692-amino acids) was discovered in 2003. It plays a major role in LDL receptor degradation and is a prominent modulator in low-density lipoprotein cholesterol (LDL-C) metabolism. PCSK9 inhibitors are monoclonal antibodies that target PCSK9 protein in liver and inhibiting this protein leads to drastically lowering harmful LDL-C level in the bloodstream. Despite widespread use of the statin, not all the high-risk patients were able to achieve targeted level of LDL-C. Using PCSK9 inhibitors could lead to a substantial decrement in LDL-C plasma level ranging from $50 \%$ to $70 \%$, either as a monotherapy or on top of statins. A large number of trials have shown robust reduction of LDL-C plasma level with the use of PCSK9 inhibitors as a monotherapy or in combination with statins in familial and nonfamilial forms of hypercholesterolemia. Moreover, PCSK9 inhibitors do not appear to increase the risk of hepatic and muscle-related side effects. PCSK9 inhibitors proved to be a highly potent and promising antihypercholesterolemic drug by decreasing LDL-R lysosomal degradation by PCSK9 protein. Statin drugs are known to have some pleiotropic effects. In this article, we are also focusing on the effects of PCSK9 inhibitor beyond LDL-C reduction like endothelial inflammation, atherosclerosis, its safety in patients with diabetes, obesity, and chronic kidney disease, and its influence on neurocognition and stroke.
\end{abstract}

\section{Introduction}

Heart disease is the leading cause of death in the US (23.7\% of total deaths in 2011) [1]. Approximately one out of three Americans died of heart disease and stroke [2]. People with high cholesterol level are twice more likely to be suffering from heart disease than normal adults. 73.7 million or $31.7 \%$ of US adults are found to have high LDL-C. Currently, near about half of the adults (48.1\%) with elevated LDLC is getting treatment. Less than one-third $(29.5 \%)$ of the population with high LDL-C is under control [1]. Familial hypercholesterolemia $(\mathrm{FH})$ which is due to the mutation of specific LDL receptor gene has been found in 1 in 299 population in the US [3]. In the case of homozygous $\mathrm{FH}$, the cholesterol level can be elevated even up to $1000 \mathrm{mg} / \mathrm{dl}$ (with LDL-C $>600 \mathrm{mg} / \mathrm{dL}$ ) and in heterozygous FH this level may reach up to $350-550 \mathrm{mg} / \mathrm{dl}$ (with LDL-C $=200-400 \mathrm{mg} / \mathrm{dL}$ ). Patients with untreated $\mathrm{FH}$ are prone to develop widespread atherosclerosis from their early life. Most of the untreated homozygous FH patients usually develop heart attack in their late teens and about half of the heterozygous FH suffer from heart disease at around 45 years for men and 55 to 60 years for females [4, 5]. According to 2013 AHA/ACC guidelines individuals with LDL-C level more than $190 \mathrm{mg} / \mathrm{dl}$ require 
high-intensity statin therapy to achieve $50 \%$ reduction. It is noteworthy that maximally tolerated dose of statin even with the combination of other nonstatin cholesterol-lowering medications is not sufficient to attain this goal, particularly in the case of $\mathrm{FH}$ [6]. In a study only $21 \%$ of patients achieved the target LDL-C level with the use of statin as a single agent [7] and a data from the UK showed among patients using combination therapy (statin and ezetimibe) only $44 \%$ patients achieved the target LDL-C level [8].

\section{Existing Lipid-Lowering Agents}

The primary lipid-lowering agents include the statin, ezetimibe, bile acid sequestrants, nicotinic acid, and fibrates. Among them, statin, ezetimibe, and bile acid sequestrants are mainly used to lower LDL-C level. Statin acts by inhibition of HMG-CoA reductase, thereby increasing LDL receptor activity. Ezetimibe inhibits cholesterol absorption by inhibiting Niemann-Pick C1-like 1 protein. Nicotinic acid and fibrates are popularly known for their triglyceride reducing property [5]. Statin is widely used to lower LDL-C and thus for primary and secondary prevention of cardiovascular disease. But this effect does not come without any side effect. Hepatic dysfunction (seen in 0.5 to $3.0 \%$ of patients) [9], myopathy (approximately $0.1 \%$ of patients develop myopathy) [10], myositis and rhabdomyolysis (near about $5 \%$ patients develop statin-associated muscle symptoms) [11], proteinuria, acute kidney injury [12], cognitive changes [13], induction of diabetes mellitus, rare cases of neuropathy [14], and druginduced lupus have been reported [9]. In the US, the statin is considered as category $\mathrm{X}$ in pregnancy [9]. Overall statin intolerance is seen approximately in $10-15 \%$ of patients in clinical practice [15]. Statin is not sufficiently useful in patients with very high plasma levels of LDL-C including $\mathrm{FH}$ patients and patients with elevated plasma levels of lipoprotein(a) even with combination with ezetimibe. Most of the cases are due to statin intolerance or their LDL-C levels are too high to control with statin-dependent therapy. So there is a pressing need to think beyond statin in such patients.

\section{Newer Hypolipidemic Drugs Either Approved Recently Or in Late Stage Development}

Recently several new classes of lipid-lowering drugs have been evolved.

Lomitapide, approved by the FDA in December 2012, is an inhibitor of microsomal triglyceride transfer protein (MTP). It is used orally and indicated mainly in homozygous $\mathrm{FH}$ or severe heterozygous $\mathrm{FH}[16]$.

Mipomersen (inhibitor of apolipoprotein B-100), an antisense oligonucleotide complementary to the coding region of human apo-B mRNA, was approved by the FDA in January 2013. It is used subcutaneously in FH patients mainly [16].

Inhibitors of cholesteryl-ester transfer protein (CETP) causes increase HDL and decrease LDL-C by 40-45\% [17]. According to REVEAL trial on anacetrapib, use of CETP inhibitors in the patient with atherosclerotic vascular disease along with intensive statin regime resulted in lower incidence of major coronary events compared to the placebo arm [18]. Bempedoic acid (ETC-1002), a novel small molecule, is known to affect carbohydrate and lipid metabolism and reduce the LDL-C level near about 27\% [19].

Proprotein convertase subtilisin/kexin type-9 (PCSK9) inhibitors are monoclonal antibodies which bind to the PCSK9 protein and regulate LDL-C level in blood. Alirocumab (Praluent, marketed by Sanofi-Aventis) and evolocumab (Repatha, sold by Amgen Inc.) have been approved by USFDA in July and August 2015, respectively. Bococizumab (RN316, Pfizer) was undergoing cardiovascular safety trial, and, after showing inadequate results, the trials have been stopped, and Pfizer also discontinued its production [20].

\section{PCSK9 and Cholesterol Pathway in the Body}

The discovery of proprotein convertase subtilisin kexin 9 (PCSK9) by Abifadel et al. in the year 2003 has revolutionized the management of $\mathrm{FH}$ and subject not responding to statins regime [21]. PCSK9 gene is located on chromosome 1p32.3 [22]. PCSK9, a serine protease, is mainly produced by the liver, intestine, and kidney. PCSK9 is synthesized as 692amino acid protein (73-kDa zymogen). After intramolecular autocatalytic processing in the endoplasmic reticulum (ER) $73-\mathrm{kDa}$ zymogen gives rise to a $14-\mathrm{kDa}$ prodomain and a $63 \mathrm{kDa}$ mature PCSK9 [23]. Under normal circumstances, the binding of LDL to its receptor (LDL-R) is followed by the endocytosis of the complex by endosomes. At the plasma membrane, PCSK9 interacts with the LDL-R, but the neutral $\mathrm{pH}$ negatively modulates this interaction. On the contrary, the acidic $\mathrm{pH}$ of the endosome increases the affinity of the two by manifolds. Consequently, the positively charged C-terminal domain of PCSK9 binds to the negatively charged ligand-binding domain of the LDL$\mathrm{R}$ [24-26] and thereby locks the LDL-R in an open conformation. The failure to attain a closed conformation in the endosome prevents normal recycling of the LDL-R to the plasma membrane. The LDL- $\mathrm{R}$ is then routed to lysosomes for degradation [24, 27]. As a consequence of decreased recycling, LDL-R at the cell surface is attenuated, and so does the LDL-C clearance. This normal physiology is magnified by gain-of-function mutations of PCSK9 leading to elevated LDL-C level and cardiovascular disease (CVD). Loss-of-function mutations of the PCSK9 result in increased surface LDL-R and improved LDL-C clearance [28].

The inverse relation between PCSK9 activity levels and LDL-R suggests that PCSK9 inhibition could have a synergistic effect with statins on LDL-C.

PCSK9 gene mutation is implicated in approximately 1$2 \%$ of patients with FH. PCSK9 gene mutation is the third commonest cause of $\mathrm{FH}$, after LDL receptor or apolipoprotein $\mathrm{B}$ (ApoB) genes mutation [29]. The loss-of-function mutation of PCSK9 gene exhibited mitigation of CVD risk by $88 \%$ in the black population [30]. This observation fueled the 
concept that PCSK9 inhibitor might be beneficial in cases of $\mathrm{FH}$ and CVD.

\section{Why PCSK9 Inhibitors Have Great Potential?}

Patient population with FH is relatively small. The prevalence of heterozygous $\mathrm{FH}$ and homozygous $\mathrm{FH}$ due to loss of function of various gene are estimated as 1 in 500 population and 1 in 1 million, respectively [5].

However, some patients who are intolerant of statin treatment as high as 3 million (or up to $15 \%$ of patients taking statins) [31]. Till now vitamin and minerals like coenzymeQ10 supplementation do not appear to prevent statin-induced muscular problems [32].

The number of the patients on statins but not achieving the target LDL-C levels would be even higher (only about one-third patient will achieve target LDL-C $<70 \mathrm{mg} / \mathrm{dl}$ even with high dose statin) [33]. In statin-treated patients there is upregulation of PCSK9 which attenuates the efficacy of statin. This observation led to the development of PCSK9 inhibitor [34]. On the other hand, PCSK9 inhibitor lowers LDL level in a dose-dependent manner. It reduces LDL-C by 70 percent and 60 percent in statin naïve patients and patients currently on statin therapy, respectively [35]. This reduction of LDL-C has been proven to have significant benefits in clinical studies irrespective of baseline cardiovascular risks.

FOURIER trial on evolocumab, a randomized, doubleblind, placebo-controlled trial enrolling 27,564 participants with history of atherosclerotic disease, has shown a substantial reduction of all cause of mortality, cardiovascular mortality, and myocardial infarction with use of evolocumab on a background of statin therapy [36]. Heightened risk of major adverse cardiac (MACE) and limb events (MALE) typically present in patient with symptomatic peripheral arterial disease. As per the results of subanalysis study of FOURIER trial, evolocumab also mitigates the risk of MALE and the relation between achieved LDL-C and lower limb events are directly proportional. Thus, reduction of LDL-C to extremely low level should be considered in a subjects with PAD, regardless of history of MI or stroke, to diminish the chance of MACE and MALE [37].

\section{A Brief Description of PCSK9 Inhibitors}

Alirocumab (approximate molecular weight of $146 \mathrm{kDa}$ ), a human monoclonal antibody (IgG1), consists of two disulfide-linked heavy chains which are disulfide-linked to a light chain $[38,39]$. It is used at a dose of $75-150 \mathrm{mg}$ subcutaneously once every two weeks. The onset of action is 4-6 hours, and elimination half-life is usually 17-20 days. It undergoes proteolysis in many tissues to form polypeptides and amino acids [40].

Evolocumab (approximate molecular weight of $141.8 \mathrm{kDa}$ ), a human monoclonal antibody (IgG2) lambda with gamma 2 heavy chain linked by a disulfide bond to lambda light chain. It is administered subcutaneously at a dose of $140 \mathrm{mg}$ every two weeks or $420 \mathrm{mg}$ once monthly. The onset of action is within 4 hours and half-life of elimination is $11-17$ days. It is metabolized by nonsaturable proteolysis $[41,42]$.

Bococizumab (approximate molecular weight of $145.1 \mathrm{kDa})$ is a humanized monoclonal antibody IgG2Kappa with gamma 2 heavy chain linked by disulfide bond with kappa light chain. It is administered at a dose of $150 \mathrm{mg}$ every two weeks or $300 \mathrm{mg}$ once monthly by subcutaneous injection [43].

\section{Brief Preclinical Studies}

In mice lack of PCSK9 is protective against atherosclerosis and overexpression of it causes increased accumulation of cholesteryl-esters in aorta leading to accelerated atherosclerosis [44]. Alirocumab reduced atherosclerosis lesion size, monocyte and T-cell recruitment, smooth muscle cells proliferation, collagen, and macrophage content, thus improving plaque morphology in mice [45]. Infection and inflammation play a key role in the expression of PCSK9 in mice model. Clearance of LPS requires LDL or HDL binding as transport protein, and PCSK9 inhibition leads to increase expression of LDL-R causing more clearance of LDL along with LPS. This finding highlights the antiinflammatory action of PCSK9 inhibitors [46]. Preclinical studies by Walley et al. and Dwivedi et al. also supported the anti-inflammatory PCSK9 inhibitors in mice animal model $[47,48]$. Alirocumab administration in mice has shown reduced circulating neutrophil, monocytes, and decreased expression of endothelial ICAM-1. Thus it attenuates monocytes attachment to vascular endothelium and dampens vascular inflammation. Food intake, body weight, and weight of the liver were unaltered with alirocumab therapy in mice [45]. Reversible liver parenchymal hypertrophy and nonsignificant adrenal cortex hypertrophy have been reported in animals. There are no increased risks of hepatitis-C virus infection, immunosuppression, neurocognitive dysfunction, type 2 diabetes mellitus, and no increased bile acid concentration in intestine, thus possessing no significant increased risk of the intestinal tumor in the animal model [49]. In rats, bococizumab administration demonstrates no adverse effects on embryo-fetal development even in dose greater than usual clinical dosage [50].

\section{Clinical Development of PCSK9 Inhibitors}

8.1. Alirocumab. Alirocumab is a novel PCSK9 inhibitor. Many studies have been done and still going on to find out its efficacy and safety profile which have been summarized in Table 1. Its LDL lowering effect is also independent of the site of injection [51].

Also, a pooled data from 3 double-blind, randomized, placebo-controlled, phase 2 studies showed alirocumab to reduce LDL-apo ${ }^{\circledR}$ and $\mathrm{Lp}(\mathrm{a})$ significantly from baseline in comparison to placebo [52]. As we know that lipoprotein is an 


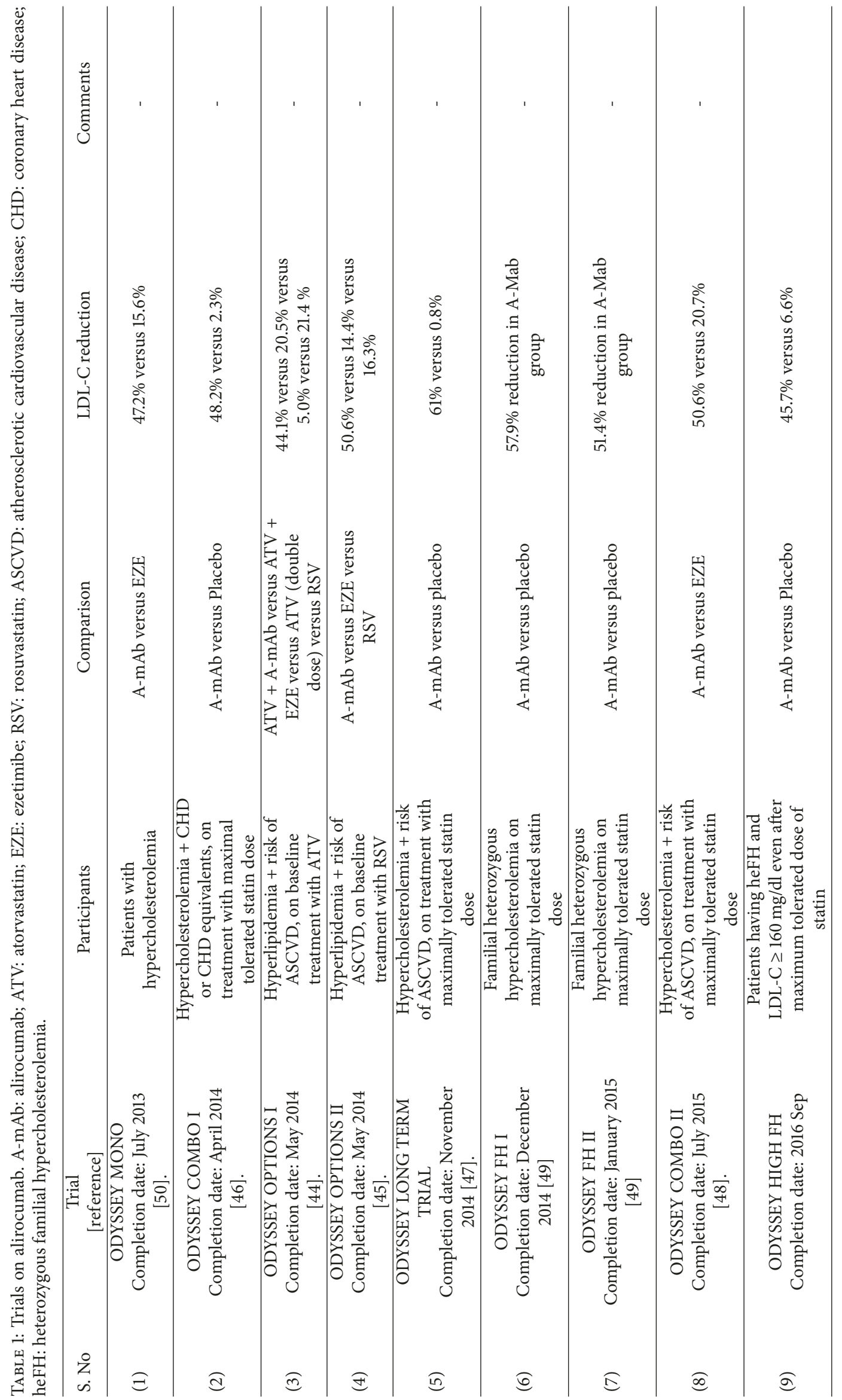




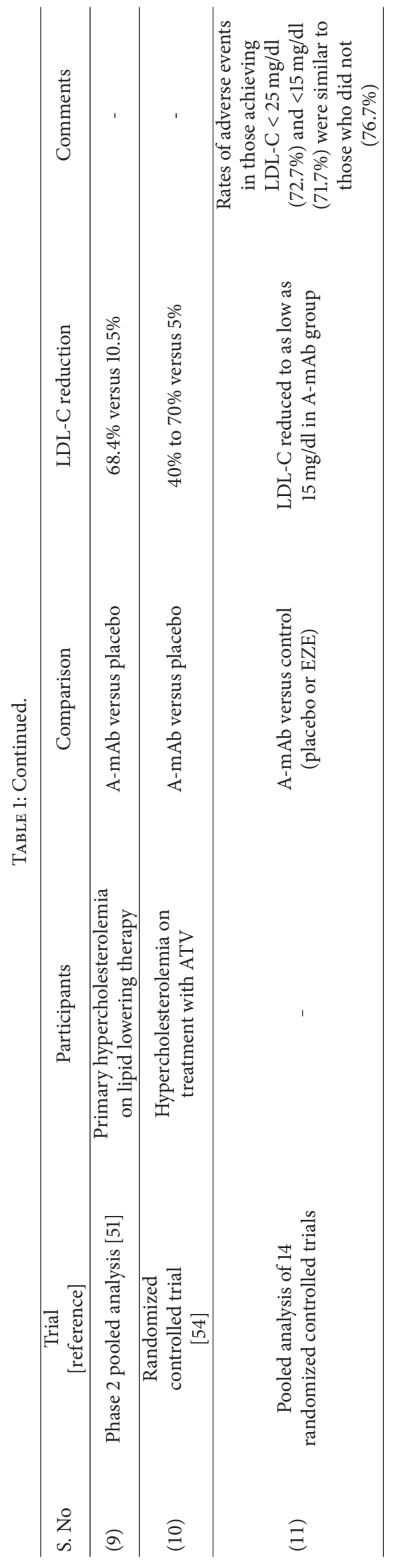


independent risk factor for CAD, this finding holds promise [53].

8.2. Evolocumab. Several clinical studies have established the efficacy and safety of evolocumab [Table 2]. GAUSS3 Randomized Clinical Trial also proved its tolerability in patients with muscle-related statin intolerance [54].

8.3. Bococizumab. Despite the fact that the initial studies showed promising result [Table 3], recently SPIRE trials showed attenuation of the effect of bococizumab in 15-20\% of patients due to the formation of antibody against its murine component. This led to the interruption of further development of this drug [55].

\section{The Role of PCSK9 beyond LDL-C Lowering}

PCSK9 inhibitors after being established as a valid option, now its effects on inflammation, endothelial function, atherosclerosis, diabetes, and obesity are now actively investigated.

(i) The PCSK9 Level in CKD Patients and HD Patients. In a study, it was shown that serum PCSK9 level was decreased in chronic kidney disease patients who were on hemodialysis (CLD-HD) and PCSK9 had a positive correlation with LDL$\mathrm{C}$ level. This signifies that PCSK9 plays a major role in regulating $\mathrm{LDL}-\mathrm{C}$ even in $\mathrm{CKD}-\mathrm{HD}$ patients. PCSK9 also is involved in the metabolism of triglyceride-rich lipoproteins in CKD-HD patients [56]. PCSK9 level tends to rise in patients with nephrotic syndrome, and it has a positive correlation with proteinuria. The PCSK9 level is also higher in patients on peritoneal dialysis in comparison to hemodialysis or renal transplant patients [57].

(ii) PCSK9 and Lipoprotein A. Lp (a) is a widely accepted cardiovascular risk factor, except for regular extracorporeal lipoprotein apheresis which is the only available modality to reduce $\mathrm{Lp}$ (a). The possible mechanism of Lp (a) reduction with use of PCSK9 inhibitors is because of the enormous expression of LDL-R due to PCSK9 inhibition, which unmasks clearance mechanism of $\operatorname{Lp}$ (a) by abundant hepatic LDL-R [58]. Additionally, Canuel et al. have reported that PCSK9 degrades LDL related protein-1, which catabolizes $\mathrm{Lp}(\mathrm{a})$. Thus, the inhibition of PCSK9 increases the catabolism of $\operatorname{Lp}(\mathrm{a})$ [59].

Alirocumab also reduces non-HDL cholesterol and fasting triglycerides significantly. Alirocumab also increases the HDL level and apolipoprotein A-1. Though the possible mechanism which increases HDL-C is not entirely clear, one likely hypothesis is that reduction of LDL-C causes reduction in cholesteryl-ester transfer protein activity, as less LDL-C is available to transfer out cholesterol from HDL particles. Eventually, it causes a relatively high level of HDL-C [60].

(iii) PCSK9 Inhibitors on Inflammation and Atherosclerosis. Atherosclerosis is a chronic inflammatory process within the arterial wall. Proinflammatory effect of PCSK9 has been shown in different experimental models. This effect is supposed to be responsible for promoting atherosclerosis independent of LDL-C level. Vascular smooth muscle cells (VSMC), oxidized LDL-C, have shown a high level of PCSK9 expression [61].

LOX-1, a receptor for oxidized LDL in VSMC, is upregulated in inflammation. It has been reported that PCSK9 stimulates transcription of LOX-1 and LOX-1, in turn, and stimulates PCSK9 expression, which facilitates atherogenesis [62]. The interaction between PCSK9 and LDL-R favors the entry of inflammatory monocytes into the arterial wall and thus promotes atherosclerosis [63]. Though it had been shown in a study that there is no effect of PCSK9 inhibitors on hs-CRP concentration level [64], the relationship of PCSK9 with systemic inflammation cannot be denied. Walley et al. revealed that loss of PCSK9 function in both mice model and human enhances pathogen lipid clearance with the help of LDL receptor and regulates inflammatory response in septic shock with better survival [48].

ATHEROREMO-IVUS study conducted by Cheng et al. revealed a linear correlation between PCSK9 level and the amount of necrotic tissue in atherosclerotic plaque [65]. Lowering of LDL-C by PCSK9 inhibitors reduces inflammation, endothelial apoptosis, and the concentration of oxidized LDL-C within the plaque. This alters the composition of the plaque more favorably. GLAGOV Randomized Clinical Trial ( $n=968$ ), which was concluded last year, revealed that addition of evolocumab to statin-treated patient causes decrease in percent atheroma plaque volume assessed by sequential intravascular ultrasound after 76 weeks of therapy [66]. Macrophage recycles the cell membrane lipid from the dead cells including RBC. When recycling capacity of macrophages exceeds, those cell membrane lipids accumulate as atheroma. Macrophage fat catabolism capacity is associated with underlying atherosclerosis, and this could be quantified by accumulation acyl-carnitine intermediates in ECF which is the direct parameter of the adequacy of betaoxidation to recycle membrane fatty acid. A study conducted by Blair et al. revealed that minimizing macrophage fat overload by reducing fat metabolism rate is favorable, which could be achieved by using statin and the newer PCSK9 inhibitors [67].

(iv) PCSK9 in Diabetic Patients. Diabetes is the major wellestablished risk factor for cardiovascular diseases. Diabetes increases the risk for atherosclerosis due to endothelial inflammation. Inflammation in blood vessels is one of the primary drivers for atherosclerosis and diabetes makes it much worse. A study conducted by Sattar et al. revealed that PCSK9 inhibitor markedly decreases atherogenic lipoproteins in diabetic patients and results are similar as seen in nondiabetic patients [68]. They did not alter the normal glucose homeostasis. Arsenault and colleagues have put the light on the fact that serum PCSK9 protein level is higher in insulin-resistant subjects, which also supports its temporal association of hyperlipidemia in diabetic patients [69]. The results from FOURIER trial demonstrated no significant difference in new-onset diabetes and neurocognitive events between evolocumab and placebo arm [36]. They reported 


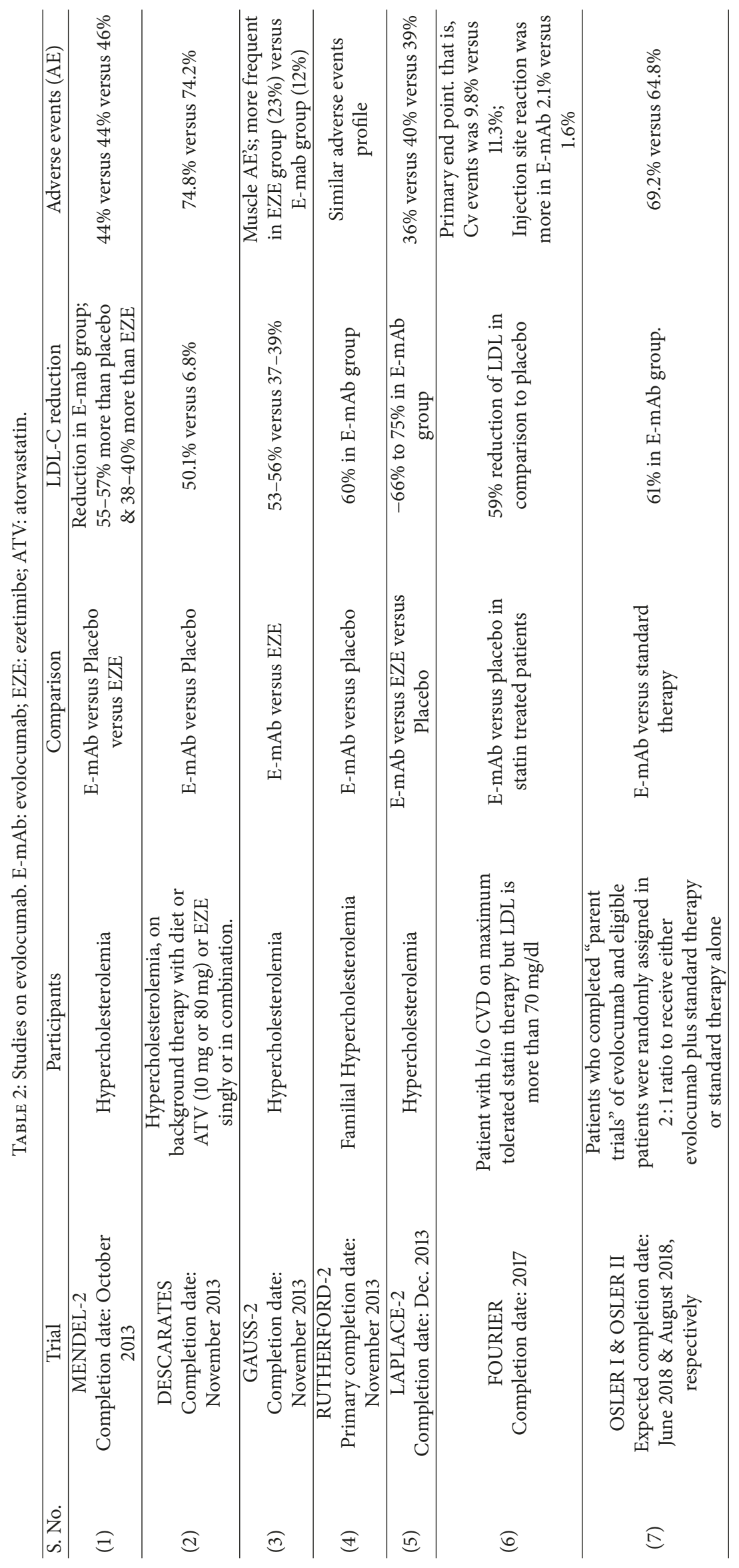




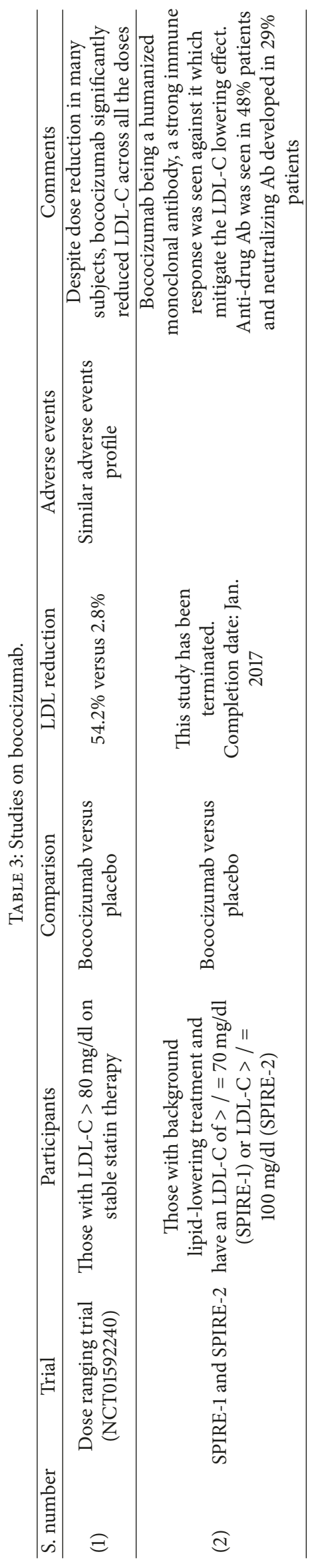


similar levels of HbAlc and FPG between both the groups in patients with diabetes, prediabetes, and normoglycemia [70].

(v) PCSK9 and Its Relation with Vitamin E, Cortisol, Adrenocorticotropic Hormone, and Gonadal Hormones. Vitamin E is one of the important antioxidants which prevent oxidative damage of long-chain PUFA and cell membrane disruption. The function of vitamin $\mathrm{E}$ transport and steroidogenesis are intricately related to LDL-C metabolism. In a recent study, 901 patients with LDL-C $\geq 2.0 \mathrm{mmol} / \mathrm{L}$ were randomly assigned to monthly subcutaneous evolocumab for 52 weeks and placebo. In evolocumab-treated substudy group, the level of vitamin $\mathrm{E}$ in LDL-C was decreased substantially, and vitamin E level in HDL was increased significantly. Cortisol level was elevated in evolocumab-treated patients, but there were no changes in ACTH, cortisol: ACTH ratio, and gonadal hormone levels. So from this above data, we can conclude that in spite of lowering LDL-C level, evolocumab does not reduce the cholesterol normalized level of vitamin E, ACTH, and gonadal hormones [71].

(vi) PCSK9 in Ischemic Stroke. PCSK9 and LDL receptor are involved in mouse brain development. After 24 to $72 \mathrm{hrs}$. of reperfusion in ischemic stroke, PCSK9 is upregulated in the dentate gyrus of the mouse model but without affecting de novo neurogenesis. PCSK9 degrades the LDL receptor in the brain (telencephalon and cerebellum) both during development and ischemia/reperfusion. PCSK9 is expressed only in the olfactory peduncle in adult mice, but it does not degrade LDL receptor. In adult mice, blood-brain barrier is impermeable to PCSK9 which explains the absence of LDL receptor lowering effect of PCSK9 in the brain of adult mice. The effect of LDL receptor in the brain is still not clear. LDL receptor-negative mice show impaired learning and memory. Though our understanding of the effects of LDL receptor excess in the brain is still evolving, ablation of the PCSK9 gene in mice did not reveal any significant effect of decreased PCSK9 level on brain recovery after an ischemic stroke. So we can hope that PCSK9 inhibition by monoclonal antibody should not hamper brain recovery after an ischemic insult [72]. A meta-analysis involving 11 studies showed there is no increased incidence of stroke with its use [73].

(vii) Effect of PCSK9 beyond Liver. PCSK9 effect beyond LDL receptor degradation in the liver is largely unknown. PCSK9 is expressed by extrahepatic tissues such as intestine, pancreas, kidney, smooth vascular cells, endothelial cells, goblet cells, and brain. In insulin-resistant diabetic patient, PCSK9 level is significantly low in duodenum compared to insulin sensitive obese patient undergoing bariatric surgery. But it remains to conclude whether insulin induces expression of PCSK9 in the intestine as it does in the liver. PCSK9-deficient mice had sevenfold increased LDL receptor expression in intestine. PCSK9-deficient mice showed reduced postprandial hypertriglyceridemia due to reduced level of ApoB. A route of cholesterol excretion that is upregulated in the PCSK9-deficient mice leads to fecal cholesterol excretion. It is also involved in nephrogenesis and binds with amiloride-sensitive epithelial sodium channel (ENaC) and mediates their degradation by proteasome pathways. PCSK9 downregulates the LDL receptor expression on the surface of the isolated human pancreatic beta cell and in PCSK9 (-I-) mice; there were increased cell surface LDL receptors. But there is an inconsistency about the deleterious effect of LDL-C accumulation in beta cells insulin secretion of the PCSK9 (-/-) mice model. This discrepancy can be explained by the PCSK9 inhibitor-mediated reduction of LDL-C level which counterbalances the deleterious effect of LDL-C accumulation inside the beta cells [74].

(viii) PCSK9 and Neurocognitive Effect. In 2012, FDA issued a warning for all statin drugs: "ill-defined memory loss or impairment" [75]. The PCSK9 inhibitor is one of the most potent and promising new therapies to lower LDL-C level nowadays. So it is imperative to discuss any plausible role of it in causing neurocognitive impairment. LDL$\mathrm{R}$ also is expressed in the brain and helps in clearing apolipoprotein-E, which is responsible for the formation of amyloid- $\beta$ which accumulates in the brain of Alzheimer's disease patient. PCSK9 gene deleted mice have shown reduced apolipoprotein-E and amyloid- $\beta$ formation. PCSK9inhibitors cannot cross blood-brain barrier in human. Longterm LDL-C lowering by PCSK9 improves arterial health which in turn protects against the development of dementia. Though there are some studies which signal to unfavorable effects on neurocognition, recently Robinson et al. showed no increased neurocognitive risk in a pooled analysis of 14 trials on PCSK9 inhibitors even after attaining an extremely low LDL-C level [76]. Loss of function of PCSK9 is not associated with any symptoms of mental retardation as well.

Currently, few trials are ongoing to search any adverse neurocognitive effect of PCSK9 inhibitors, and we have to wait until the end of 2017-2018 for a final opinion regarding this aspect.

(ix) Available Safety Data on PCSK9 Inhibitors. The most common adverse events occurring in alirocumab treated patients were gastrointestinal disorders, infections and infestations, musculoskeletal disorders, and skin and subcutaneous tissue disorders [77]. One patient with the history of atrial fibrillation and chronic obstructive pulmonary disorder had a pulmonary embolism after alirocumab therapy in ODYSSEY MONO study [78].

Nasopharyngitis, upper respiratory tract infection, influenza, and back pain are commonly found adverse effects regarding the use of evolocumab [79]. The increment of creatine kinase levels to more than five times the ULN occurred in $1.2 \%$ of patients in the evolocumab group in DESCARTES Trial [80]. Acute pancreatitis has been reported in MENDEL 2 trial as well [81].

Upper respiratory tract infection, nasopharyngitis, diarrhea, urinary tract infection, arthralgia, bronchitis, injection site erythema, gastroesophageal reflux disease, and cough are commonly found adverse effects after bococizumab use [82].

Interestingly, PCSK9 inhibitors do not lead to an increased rate of new-onset diabetes [83]. Though high blood glucose during the treatment period and baseline 
HbAlc of more than $6.5 \%$ have been found in alirocumab treated patients, there was no pattern in changes in either blood glucose or HbAlc from screening to week 24 [84]. Evolocumab treatment did not show any adverse effect on glycemic measures in a 52-week placebo-controlled trial [80].

A meta-analysis of 25 randomized controlled trials has shown no significant difference regarding the occurrence of adverse events between PCSK9 inhibitor group and placebo group (or ezetimibe group) [85].

Cholesterol is an important component of myelin protein. So, lipid-lowering therapies may play a role to hamper the neural structure and function. Along with that reduced serum cholesterol may also enhance the blood-brain barrier permeability. It may result in increased exposure of the central nervous system to the toxins in the blood. So there is a concern whether PCSK9 inhibitors can cause cognitive dysfunction by lowering cholesterol level [75]. A metaanalysis has shown a significant increase in neurocognitive events with PCSK9 inhibitor therapy in comparison with placebo therapy. But the analysis has some limitations due to lack of uniform data, heterogeneity of the studies, and lack of uniform definitions of the cardiovascular events [86]. On the other hand, LDL receptor causes clearance of apolipoprotein-E, a protein responsible for Alzheimer's disease. So PCSK9 inhibitors may have the protective role for this disease. Long-term PCSK9 inhibitor therapy can prevent vascular dementia by improving arterial health as well. So there may be a positive effect of PCSK9 inhibitors on neurocognitive functions. Cognitive side effects have been found as an uncommon finding in the OSLER study. Less than $1 \%$ patients showed amnesia and less than $1 \%$ patients showed mental impairment, but no precise data is available till now [75]. Apart from neurocognitive impairment, increased incidences of hemorrhagic stroke, hormonal insufficiency, and hemolytic anemia have been found to be associated with the very low LDL-C level [79]. There was only statistically insignificant increased risk of cataract in a recent analysis [76].

Pooled analysis from 10 ODYSSEY Trials established no increase adverse events in patients on alirocumab therapy [87].

Naturally more time-tested trials are required for conclusive data. To summarize, published evidence from the trials suggests that PCSK9 inhibitors are well tolerated and with good safety profile [84].

\section{Conclusion and Future Direction}

The discovery of PCSK9 proteins has changed the dynamics of lipid control in hypercholesterolemic patients. PCSK9 inhibitors pave the path of achieving an extremely low plasma level of LDL-C and have shown to reduce lifetime risk for CVD events. They are also involved in decreasing endothelial inflammation which is the key factor for atherosclerosis. Very aggressive lowering of LDL-C by PCSK9 inhibitors leads to plaque stabilization and regression. Large phase II and III trials for these monoclonal Abs have shown its safety, efficacy, and effectiveness in patients who are at risk for cardiovascular diseases due to dyslipidemia. Many trials have revealed that PCSK9 inhibitors have reduced all causes of mortality including cardiovascular mortality with less adverse effects like myopathy and hepatotoxicity. Though SPIRE trials were terminated early for the concern of immunogenicity, the immunogenicity of evolocumab is extremely low and neutralizing anti-drug antibody was seen only in $1.3 \%$ patients on alirocumab. Newer drug Inclisiran, a PCSK9-specific small interfering RNA, is also being studied. ODYSSEY OUTCOME study and other trials are also in progress to evaluate the whole spectrum of PCSK9 inhibitors, and the results are scheduled to come by the end of 2017 to 2018. It is to be evaluated by subsequent trials whether these pleiotropic effects would confer substantial morbidity and mortality benefits.

\section{Conflicts of Interest}

The authors declare that they have no conflicts of interest.

\section{References}

[1] Cholesterol Facts High, http://www.cdc.gov/cholesterol/facts .htm.March.

[2] A. S. Go, D. Mozaffarian, V. L. Roger et al., "Heart disease and stroke statistics - 2014 update: a report from the American heart association," Circulation, vol. 129, no. 3, pp. e28-e292, 2014.

[3] S. D. D. Ferranti, A. M. Rodday, M. Mendelson, J. B. Wong, L. K. Leslie, and R. C. Sheldrick, "6: What is the Prevalence of Familial Hypercholesterolemia in the US? Session Title: Lipid-Lowering Trials. Circulation.2014; 130: A19656," in Core 2. Epidemiology and Prevention of CV Disease: Physiology, Pharmacology and Lifestyle Abstract, A19656, 130, 1965.

[4] Bob Carlson, "Familial Hypercholesterolemia Captures Gene Test Controversies.Biotechnol Healthc," in Familial Hypercholesterolemia Captures Gene Test Controversies, vol. 7, p. 89, Spring, Biotechnol Healthc, 2010.

[5] G. K. Hovingh, M. H. Davidson, J. J. P. Kastelein, and A. M. O'Connor, "Diagnosis and treatment of familial hypercholesterolaemia," European Heart Journal, vol. 34, no. 13, pp. 962-971, 2013.

[6] N. J. Stone, "ACC/AHA Guideline on the Treatment of Blood Cholesterol to Reduce Atherosclerotic Cardiovascular Risk in Adults," A Report of the American College of Cardiology/American Heart Association, vol. 129, supplement 2, pp. S1S45, 2014.

[7] A. H. Pijlman, R. Huijgen, S. N. Verhagen et al., "Evaluation of cholesterol lowering treatment of patients with familial hypercholesterolemia: a large cross-sectional study in The Netherlands," Atherosclerosis, vol. 209, no. 1, pp. 189-194, 2010.

[8] Audit of the Management of Familial Hypercholesterolaemia 2010: Full Report. Royal College of Physicians website 2010, http://www.rcplondon.ac.uk/resources/audits/FH.

[9] Rosenson RS, "statins: Actions, side effects, and administration," http://www.uptodate.com/contents/statins-actions-side-effects-andadministrationsource $=$ search_result\&search $=$ statin \& selectedTitle $=1 \sim 150$.

[10] D. J. Graham, J. A. Staffa, D. Shatin et al., "Incidence of hospitalized rhabdomyolysis in patients treated with lipid-lowering drugs," The Journal of the American Medical Association, vol. 292, no. 21, pp. 2585-2590, 2004. 
[11] U. Laufs, H. Scharnagl, M. Halle, E. Windler, M. Endres, and W. März, "Treatment options for statin-associated muscle symptoms," Deutsches Ärzteblatt International, vol. 112, no. 44, pp. 748-55, 2015.

[12] A. A. Alsheikh-Ali, M. S. Ambrose, J. T. Kuvin, and R. H. Karas, "The safety of rosuvastatin as used in common clinical practice: A postmarketing analysis," Circulation, vol. 111, no. 23, pp. 30513057, 2005.

[13] D. A. Redelmeier, D. Thiruchelvam, and N. Daneman, "Delirium after elective surgery among elderly patients taking statins," Canadian Medical Association Journal, vol. 179, no. 7, pp. 645652, 2008.

[14] D. Gaist, U. Jeppesen, M. Andersen, L. A. García Rodríguez, J. Hallas, and S. H. Sindrup, "Statins and risk of polyneuropathy: A case-control study," Neurology, vol. 58, no. 9, pp. 1333-1337, 2002.

[15] M. Banach, M. Rizzo, and P. P. Toth, "Statin intolerance an attempt at a unified definition. Position paper from an international lipid expert panel," Archives of Medical Science, vol. 11, no. 1, pp. 1-23, 2015.

[16] Elena Citkowitz, "Familial Hypercholesterolemia Treatment \& Management," 2015, http://emedicine.medscape.com/article/ 121298-treatment\#d7.

[17] J. J. P. Kastelein, J. Besseling, S. Shah et al., "Anacetrapib as lipid-modifying therapy in patients with heterozygous familial hypercholesterolaemia (REALIZE): A randomised, doubleblind, placebo-controlled, phase 3 study," The Lancet, vol. 385, no. 9983, pp. 2153-2161, 2015.

[18] "Effects of Anacetrapib in Patients with Atherosclerotic Vascular Disease," The New England Journal of Medicine, vol. 377, no. 13, pp. 1217-1227, 2017.

[19] C. M. Ballantyne, M. H. Davidson, D. E. MacDougall et al., "Efficacy and safety of a novel dual modulator of adenosine triphosphate-citrate lyase and adenosine monophosphateactivated protein kinase in patients with hypercholesterolemia: Results of a multicenter, randomized, double-blind, placebocontrolled, parallel-group trial," Journal of the American College of Cardiology, vol. 62, no. 13, pp. 1154-1162, 2013.

[20] A. M. Sible, J. J. Nawarskas, and J. R. Anderson, "PCSK9 inhibitors: An innovative approach to treating hyperlipidemia," Cardiology in Review, vol. 24, no. 3, pp. 141-152, 2016.

[21] M. Abifadel, M. Varret, J. Rabès et al., "Mutations in PCSK9 cause autosomal dominant hypercholesterolemia," Nature Genetics, vol. 34, no. 2, pp. 154-156, 2003.

[22] D. Urban, J. Pöss, M. Böhm, and U. Laufs, "Targeting the proprotein convertase subtilisin/kexin type 9 for the treatment of dyslipidemia and atherosclerosis," Journal of the American College of Cardiology, vol. 62, no. 16, pp. 1401-1408, 2013.

[23] N. G. Seidah and A. Prat, "The proprotein convertases are potential targets in the treatment of dyslipidemia," Journal of Molecular Medicine, vol. 85, no. 7, pp. 685-696, 2007.

[24] P. L. Surdo, M. J. Bottomley, A. Calzetta et al., "Mechanistic implications for LDL receptor degradation from the PCSK9/LDLR structure at neutral pH," EMBO Reports, vol. 12, no. 12, pp. 1300-1305, 2011.

[25] T. Yamamoto, C. Lu, and R. O. Ryan, "A two-step binding model of PCSK9 interaction with the low density lipoprotein receptor," The Journal of Biological Chemistry, vol. 286, no. 7, pp. 5464$5470,2011$.

[26] K. Tveten, Ø. L. Holla, J. Cameron et al., "Interaction between the ligand-binding domain of the LDL receptor and the Cterminal domain of PCSK9 is required for PCSK9 to remain bound to the LDL receptor during endosomal acidification," Human Molecular Genetics, vol. 21, no. 6, Article ID ddr578, pp. 1402-1409, 2012.

[27] S. C. Blacklow, "Versatility in ligand recognition by LDL receptor family proteins: advances and frontiers," Current Opinion in Structural Biology, vol. 17, no. 4, pp. 419-426, 2007.

[28] V. Bittner, "Pleiotropic Effects of PCSK9 (Proprotein Convertase Subtilisin/Kexin Type 9) Inhibitors?" Circulation, vol. 134, no. 22, pp. 1695-1696, 2016.

[29] J. Cohen, A. Pertsemlidis, I. K. Kotowski, R. Graham, C. K. Garcia, and H. H. Hobbs, "Low LDL cholesterol in individuals of African descent resulting from frequent nonsense mutations in PCSK9," Nature Genetics, vol. 37, no. 2, pp. 161-165, 2005.

[30] N. G. Seidah, Z. Awan, M. Chrétien, and M. Mbikay, "PCSK9: A key modulator of cardiovascular health," Circulation Research, vol. 114, no. 6, pp. 1022-1036, 2014.

[31] D. H. Fitchett, R. A. Hegele, and S. Verma, "Statin intolerance," Circulation, vol. 131, no. 13, pp. e389-e391, 2015.

[32] J. M. Young, C. M. Florkowski, S. L. Molyneux et al., "Effect of Coenzyme Q10 Supplementation on Simvastatin-Induced Myalgia," American Journal of Cardiology, vol. 100, no. 9, pp. 1400-1403, 2007.

[33] D. G. Karalis, R. D. Subramanya, S. E. Hessen, L. Liu, and M. F. Victor, "Achieving optimal lipid goals in patients with coronary artery disease," American Journal of Cardiology, vol. 107, no. 6, pp. 886-890, 2011.

[34] G. D. Norata, G. Tibolla, and A. L. Catapano, "PCSK9 inhibition for the treatment of hypercholesterolemia: Promises and emerging challenges," Vascular Pharmacology, vol. 62, no. 2, pp. 103-111, 2014.

[35] Rosenson RS, "Lipid lowering with drugs other than statins and fibrates," http://www.uptodate.com/contents/lipid-loweringwith-drugs-other-than-statins-andfibrates? source=machineLearning\&search $=$ pcsk9\&selectedTitle $=1 \sim 26 \&$ anchor $=$ H370516502\&sectionRank=1\#H370516502.

[36] M. S. Sabatine, R. P. Giugliano, A. C. Keech et al., "Evolocumab and clinical outcomes in patients with cardiovascular disease," The New England Journal of Medicine, vol. 376, no. 18, pp. 17131722, 2017.

[37] M. P. Bonaca, P. Nault, R. P. Giugliano et al., "Low-Density Lipoprotein Cholesterol Lowering With Evolocumab and Outcomes in Patients With Peripheral Artery Disease," Circulation, vol. 137, no. 4, pp. 338-350, 2018.

[38] Alirocumab label Label revised,.

[39] International Nonproprietary Names for Pharmaceutical Substances (INN), "WHO Drug Information," Vol. 27, No. 1, 2013.

[40] "Alirocumab: Drug information," http://www.uptodate.com/ contents/alirocumab-drug-information?source=see_link.

[41] A. F. G. Cicero, A. Colletti, and C. Borghi, "Profile of evolocumab and its potential in the treatment of hyperlipidemia," Drug Design, Development and Therapy, vol. 9, pp. 3073-3082, 2015.

[42] "Evolocumab: Drug information," http://www.uptodate.com/ contents/evolocumab-drug-information? source=see_link.

[43] "Bococizumab," http://medcheminternational.blogspot.in/ 2015/09/bococizumab.html.

[44] M. Denis, J. Marcinkiewicz, A. Zaid et al., "Gene inactivation of proprotein convertase subtilisin/kexin type 9 reduces atherosclerosis in mice," Circulation, vol. 125, no. 7, pp. 894-901, 2012. 
[45] S. Kühnast, J. W. A. Van Der Hoorn, E. J. Pieterman et al., "Alirocumab inhibits atherosclerosis, improves the plaque morphology, and enhances the effects of a statin," Journal of Lipid Research, vol. 55, no. 10, pp. 2103-2112, 2014.

[46] K. R. Feingold, A. H. Moser, J. K. Shigenaga, S. M. Patzek, and C. Grunfeld, "Inflammation stimulates the expression of PCSK9," Biochemical and Biophysical Research Communications, vol. 374, no. 2, pp. 341-344, 2008.

[47] D. J. Dwivedi, P. M. Grin, M. Khan et al., "Differential expression of PCSK9 modulates infection, inflammation, and coagulation in a murine model of sepsis," Shock, vol. 46, no. 6, pp. 672-680, 2016.

[48] K. R. Walley, K. R. Thain, J. A. Russell et al., "PCSK9 is a critical regulator of the innate immune response and septic shock outcome," Science Translational Medicine, vol. 6, no. 258, pp. 258-ral43, 2014.

[49] "The endocrinologic and metabolic drugs advisory committee meeting. Briefing document," Praluent(alirocumab) injection, 2015, http://www.fda.gov/downloads/AdvisoryCommittees/ CommitteesMeetingMaterials/Drugs/EndocrinologicandMetabolicDrugsAdvisoryCommittee/UCM449865.pdf.

[50] S. N. Campion, B. Han, G. D. Cappon et al., "Decreased maternal and fetal cholesterol following maternal bococizumab (anti-PCSK9 monoclonal antibody) administration does not affect rat embryo-fetal development," Regulatory Toxicology and Pharmacology, vol. 73, no. 2, pp. 562-570, 2015.

[51] C. Lunven, T. Paehler, F. Poitiers et al., "A randomized study of the relative pharmacokinetics, pharmacodynamics, and safety of alirocumab, a fully human monoclonal antibody to PCSK9, after single subcutaneous administration at three different injection sites in healthy subjects," Cardiovascular Therapeutics, vol. 32, no. 6, pp. 297-301, 2014.

[52] D. Gaudet, D. J. Kereiakes, J. M. McKenney et al., "Effect of alirocumab, a monoclonal proprotein convertase subtilisin/kexin 9 antibody, on lipoprotein(a) concentrations (a pooled analysis of $150 \mathrm{mg}$ every two weeks dosing from phase 2 trials)," American Journal of Cardiology, vol. 114, no. 5, pp. 711715, 2014.

[53] D. Gaudet, G. F. Watts, J. G. Robinson et al., "Effect of Alirocumab on Lipoprotein(a) Over $\geq 1.5$ Years (from the Phase 3 ODYSSEY Program)," American Journal of Cardiology, vol. 119, no. 1 , pp. 40-46, 2017.

[54] S. E. Nissen, E. Stroes, R. E. Dent-Acosta et al., "Efficacy and tolerability of evolocumab vs ezetimibe in patients with musclerelated statin intolerance: The GAUSS-3 randomized clinical trial," Journal of the American Medical Association, vol. 315, no. 15, pp. 1580-1590, 2016.

[55] P. M. Ridker, J. Revkin, P. Amarenco et al., "Cardiovascular efficacy and safety of bococizumab in high-risk patients," The New England Journal of Medicine, vol. 376, no. 16, pp. 1527-1539, 2017.

[56] H. Abujrad, J. Mayne, M. Ruzicka et al., "Chronic kidney disease on hemodialysis is associated with decreased serum PCSK9 levels," Atherosclerosis, vol. 233, no. 1, pp. 123-129, 2014.

[57] P. Pavlakou, E. Liberopoulos, E. Dounousi, and M. Elisaf, "PCSK9 in chronic kidney disease," International Urology and Nephrology, vol. 49, no. 6, pp. 1015-1024, 2017.

[58] S. Tsimikas, "Lipoprotein(a): Novel target and emergence of novel therapies to lower cardiovascular disease risk," Current Opinion in Endocrinology, Diabetes and Obesity, vol. 23, no. 2, pp. 157-164, 2016.
[59] M. Canuel, X. Sun, M.-C. Asselin, E. Paramithiotis, A. Prat, and N. G. Seidah, "Proprotein Convertase Subtilisin/Kexin Type 9 (PCSK9) Can Mediate Degradation of the Low Density Lipoprotein Receptor-Related Protein 1 (LRP-1)," PLoS ONE, vol. 8, no. 5, Article ID e64145, 2013.

[60] T. Teramoto, M. Kobayashi, H. Tasaki et al., "Efficacy and safety of alirocumab in Japanese patients with heterozygous familial hypercholesterolemia or at high cardiovascular risk with hypercholesterolemia not adequately controlled with statins - ODYSSEY JAPAN randomized controlled trial," Circulation Journal, vol. 80, no. 9, pp. 1980-1987, 2016.

[61] L. Liberale, F. Montecucco, G. G. Camici et al., "Treatment with proprotein convertase subtilisin/kexin type 9 (PCSK9) inhibitors to reduce cardiovascular inflammation and outcomes," Current Medicinal Chemistry, vol. 24, no. 14, pp. 14031416, 2017.

[62] Z. Ding, S. Liu, X. Wang et al., "Cross-Talk between LOX-1 and PCSK9 in vascular tissues," Cardiovascular Research, vol. 107, no. 4, pp. 556-567, 2015.

[63] M. D. Shapiro and S. Fazio, "PCSK9 and atherosclerosis - lipids and beyond," Journal of Atherosclerosis and Thrombosis, vol. 24, no. 5, pp. 462-472, 2017.

[64] A. Sahebkar, P. Di Giosia, C. A. Stamerra et al., "Effect of monoclonal antibodies to PCSK9 on high-sensitivity C-reactive protein levels: A meta-analysis of 16 randomized controlled treatment arms," British Journal of Clinical Pharmacology, vol. 81, no. 6, pp. 1175-1190, 2016.

[65] J. M. Cheng, R. M. Oemrawsingh, H. M. Garcia-Garcia et al., "PCSK9 in relation to coronary plaque inflammation: Results of the ATHEROREMO-IVUS study," Atherosclerosis, vol. 248, pp. 117-122, 2016.

[66] S. J. Nicholls, R. Puri, T. Anderson et al., "Effect of evolocumab on progression of coronary disease in statin-treated patients: The GLAGOV randomized clinical trial," Journal of the American Medical Association, vol. 316, no. 22, pp. 2373-2384, 2016.

[67] H. C. Blair, J. Sepulveda, and D. J. Papachristou, "Nature and nurture in atherosclerosis: The roles of acylcarnitine and cell membrane-fatty acid intermediates," Vascular Pharmacology, vol. 78, pp. 17-23, 2016.

[68] N. Sattar, D. Preiss, J. G. Robinson et al., "Lipid-lowering efficacy of the PCSK9 inhibitor evolocumab (AMG 145) in patients with type 2 diabetes: A meta-analysis of individual patient data," The Lancet Diabetes \& Endocrinology, vol. 4, no. 5, pp. 403-410, 2016.

[69] B. J. Arsenault, E. Pelletier-Beaumont, N. Alméras et al., "PCSK9 levels in abdominally obese men: Association with cardiometabolic risk profile and effects of a one-year lifestyle modification program," Atherosclerosis, vol. 236, no. 2, pp. 321326, 2014.

[70] M. S. Sabatine, L. A. Leiter, S. D. Wiviott et al., "Cardiovascular safety and efficacy of the PCSK9 inhibitor evolocumab in patients with and without diabetes and the effect of evolocumab on glycaemia and risk of new-onset diabetes: A prespecified analysis of the FOURIER randomised controlled trial," The Lancet Diabetes \& Endocrinology, 2017.

[71] D. J. Blom, C. S. Djedjos, M. L. Monsalvo et al., "Effects of evolocumab on Vitamin E and steroid hormone levels: Results from the 52-week, phase 3, double-blind, randomized,placebocontrolled DESCARTES study," Circulation Research, vol. 117, no. 8, pp. 731-741, 2015. 
[72] E. Rousselet, J. Marcinkiewicz, J. Kriz et al., "PCSK9 reduces the protein levels of the LDL receptor in mouse brain during development and after ischemic stroke," Journal of Lipid Research, vol. 52, no. 7, pp. 1383-1391, 2011.

[73] A. R. Khan, C. Bavishi, H. Riaz et al., "Increased Risk of Adverse Neurocognitive Outcomes with Proprotein Convertase Subtilisin-Kexin Type 9 Inhibitors," Circulation: Cardiovascular Quality and Outcomes, vol. 10, no. 1, Article ID e003153, 2017.

[74] B. Cariou, K. Si-Tayeba, and C. Le Maya, "Role of PCSK9 beyond liver involvement," Current Opinion in Pediatrics, vol. 26, no. 3, pp. 155-161, 2015.

[75] K. J. Swiger and S. S. Martin, "PCSK9 inhibitors and neurocognitive adverse events: Exploring the FDA directive and a proposal for N-of-1 trials," Drug Safety, vol. 38, no. 6, pp. 519526, 2015.

[76] J. G. Robinson, R. S. Rosenson, M. Farnier et al., "Safety of Very Low Low-Density Lipoprotein Cholesterol Levels With Alirocumab: Pooled Data From Randomized Trials," Journal of the American College of Cardiology, vol. 69, no. 5, pp. 471-482, 2017.

[77] M. J. Koren, E. M. Roth, J. M. McKenney et al., "Safety and efficacy of alirocumab $150 \mathrm{mg}$ every 2 weeks, a fully human proprotein convertase subtilisin/kexin type 9 monoclonal antibody: A phase II pooled analysis," Postgraduate Medical Journal, vol. 127, no. 2, pp. 125-132, 2015.

[78] E. M. Roth and J. M. McKenney, "ODYSSEY MONO: Effect of alirocumab $75 \mathrm{mg}$ subcutaneously every 2 weeks as monotherapy versus ezetimibe over 24 weeks," Future Cardiology, vol. 11, no. 1, pp. 27-37, 2015.

[79] M. Hassan and M. Yacoub, "GAUSS-2, RUTHERFORD-2, LAPLACE-2, DESCARTES, and TESLA Part B: PCSK9 inhibitors gain momentum," Global Cardiology Science and Practice, vol. 2014, no. 4, p. 49, 2014.

[80] D. J. Blom, T. Hala, M. Bolognese et al., "A 52-week placebocontrolled trial of evolocumab in hyperlipidemia," The New England Journal of Medicine, vol. 370, no. 19, pp. 1809-1819, 2014.

[81] M. J. Koren, P. Lundqvist, M. Bolognese et al., "Anti-PCSK9 monotherapy for hypercholesterolemia: The MENDEL-2 randomized, controlled phase III clinical trial of evolocumab," Journal of the American College of Cardiology, vol. 63, no. 23, pp. 2531-2540, 2014.

[82] C. M. Ballantyne, J. Neutel, A. Cropp et al., "Results of bococizumab, a monoclonal antibody against proprotein convertase subtilisin/kexin type 9, from a randomized, placebocontrolled, dose-ranging study in statin-treated subjects with hypercholesterolemia," American Journal of Cardiology, vol. 115, no. 9, pp. 1212-1221, 2015.

[83] K. G. Parhofer, "PCSK9 inhibitors in hypercholesterolemia: New hope for patients with diabetes mellitus?" Herz, vol. 41, no. 3, pp. 217-223, 2016.

[84] I. Gouni-Berthold and H. K. Berthold, "PCSK9 antibodies for the treatment of hypercholesterolemia," Nutrients, vol. 6, no. 12, pp. 5517-5533, 2014.

[85] X.-L. Zhang, Q.-Q. Zhu, L. Zhu et al., "Safety and efficacy of anti-PCSK9 antibodies: A meta-analysis of 25 randomized, controlled trials," BMC Medicine, vol. 13, no. 1, article no. 123, 2015.

[86] M. J. Lipinski, U. Benedetto, R. O. Escarcega et al., "The impact of proprotein convertase subtilisin-kexin type 9 serine protease inhibitors on lipid levels and outcomes in patients with primary hypercholesterolaemia: A network meta-analysis," European Heart Journal, vol. 37, no. 6, pp. 536-545, 2016.
[87] K. K. Ray, H. N. Ginsberg, M. H. Davidson et al., "Reductions in Atherogenic Lipids and Major Cardiovascular Events: A Pooled Analysis of 10 ODYSSEY Trials Comparing Alirocumab with Control," Circulation, vol. 134, no. 24, pp. 1931-1943, 2016. 


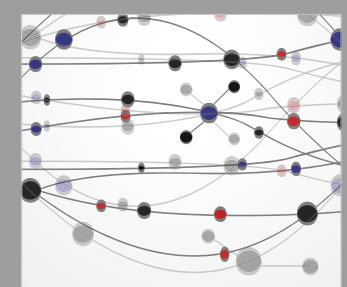

The Scientific World Journal
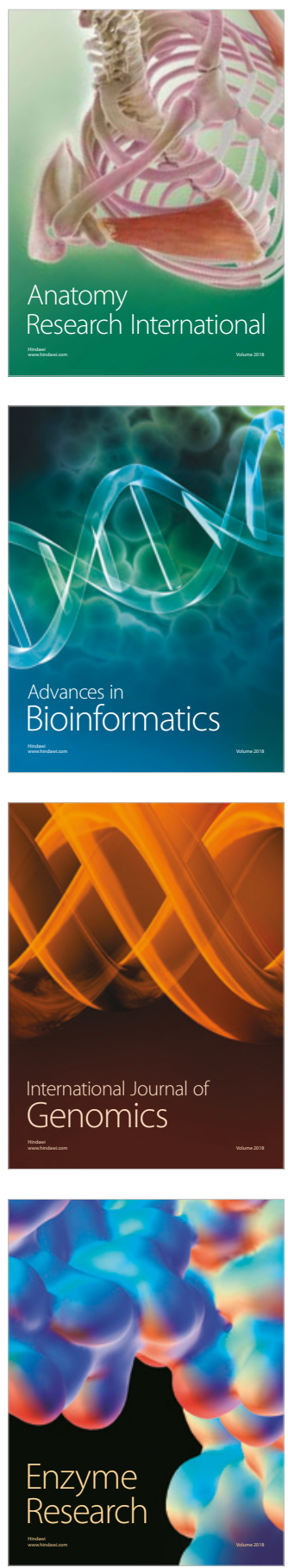
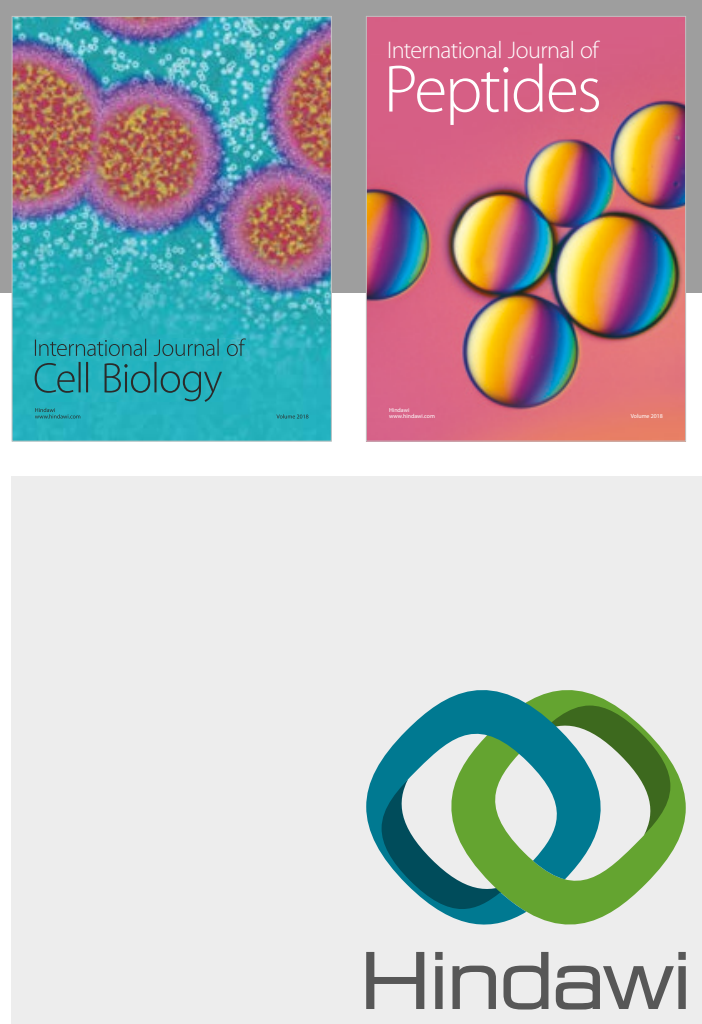

Submit your manuscripts at

www.hindawi.com
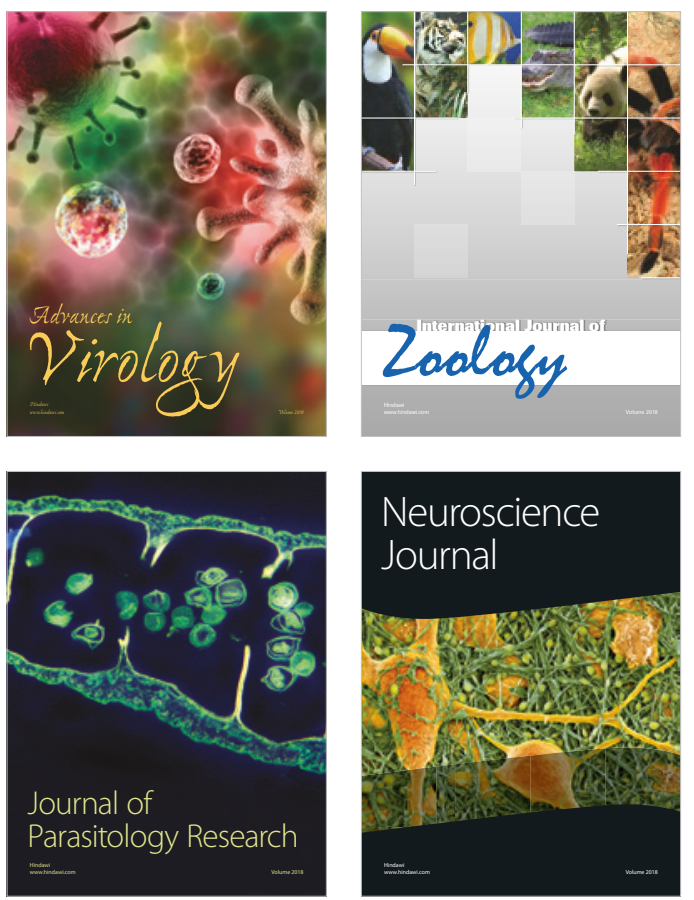
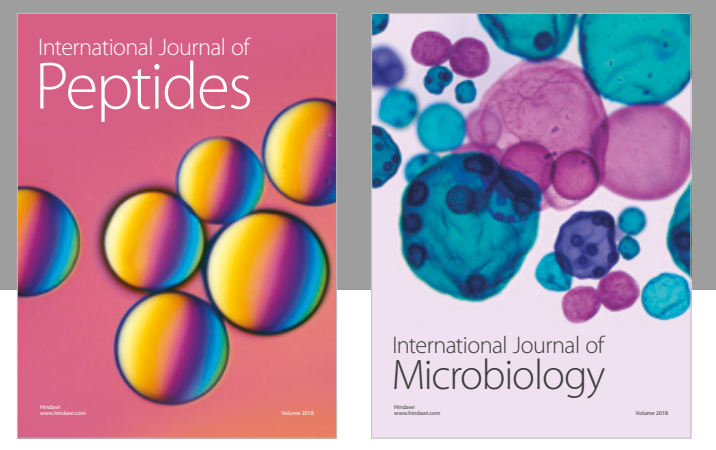

nternational Journal of Microbiology
Journal of
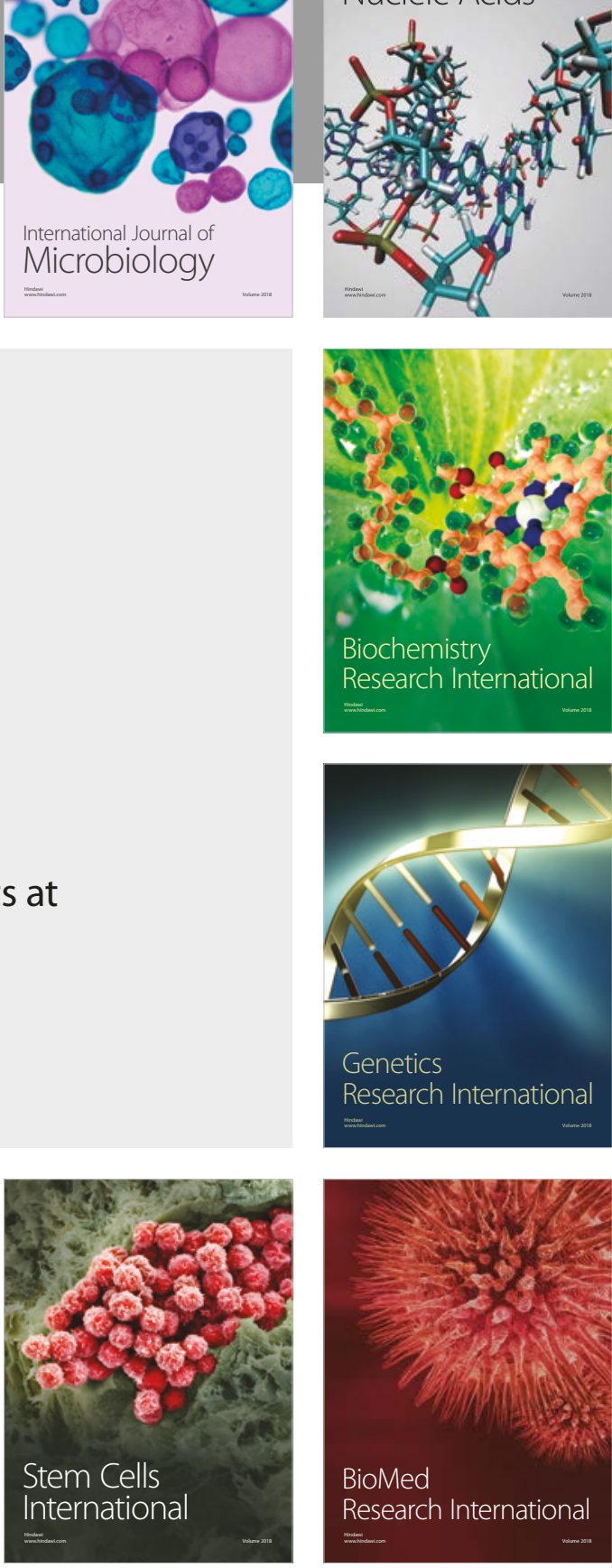
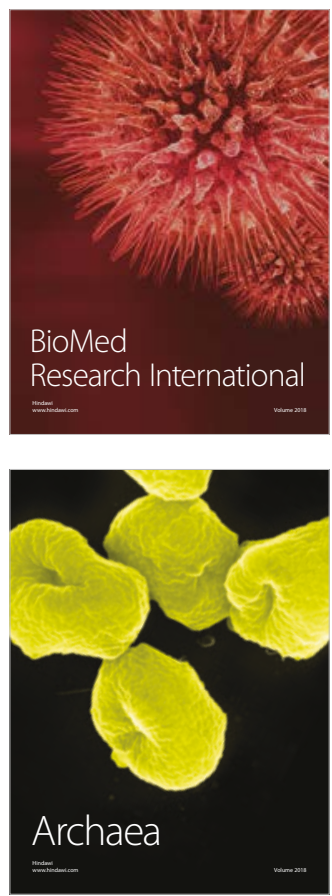